Mucormycoses

rhino-orbito-

cérébrales

\section{Traitement chirurgical,} état de l'art

(ROC) constituent une infection fongique grave potentiellement mortelle. Ces infections surviennent le plus souvent chez des patients immunodéprimés et peuvent être rapidement évolutives. La chirurgie de résection des lésions nécrotiques a montré son intérêt en termes de survie dans les mucormycoses de localisation pulmonaire et cutanée. Cependant, le traitement des mucormycoses de localisation rhino-orbitocérébrale n'est pas défini en fonction de l'étendue de la maladie alors que les voies d'extension de cette pathologie sont bien connues. La chirurgie endonasale par voie endoscopique dans cette indication permet le contrôle local de l'infection, de meilleures suites postopératoires que la chirurgie par les voies transfaciales et diminue le coût esthétique des séquelles. Toutefois, dans certains cas cette chirurgie est associée à des abords externes pour permettre une résection cutanée, endobuccale ou une exentération dont les indications restent toujours discutées. L'étude rétrospective de 22 patients présentant une mucormycose ROC issus de l'étude Rétrozygo a permis de montrer que le traitement chirurgical radical permet un contrôle local de la maladie et qu'il est associé à une survie améliorée. La validation de ces données nécessite une étude prospective (PHRC MICCA, en cours) qui permettra ainsi d'uniformiser la prise en charge de cette pathologie rare mais gravissime. <

Alors que le traitement chirurgical des mucormycoses rhino-orbito-cérébrales est rapporté par de nombreux auteurs [1-10], l'extension de la résection chirurgicale reste débattue. Certains auteurs prônent la simple résection des tissus nécrotiques alors que d'autres sont favorables à l'obtention d'un contrôle local de la mala-

\section{Pierre Vironneau, Benjamin Verillaud, Hugo Tran, Khaled Altabaa, Jean-Philippe Blancal, Élisabeth Sauvaget, Philippe Herman, Romain Kania}

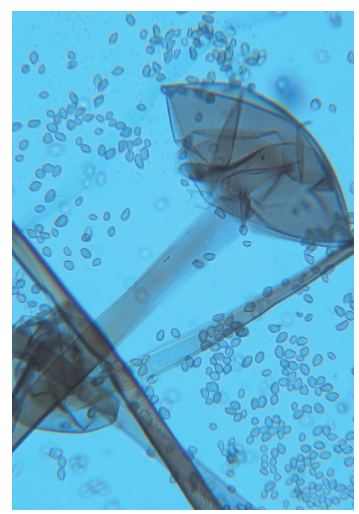

Service d'otorhinolaryngologie et de chirurgie cervico-faciale, Hôpital Lariboisière, AP-HP, Paris, Université Paris 7, Paris Sorbonne Cité, 2, rue Ambroise Paré, 75475 Paris Cedex 10, France. romain.kania@lrb.aphp.fr

die par la chirurgie. Selon cette dernière modalité, la chirurgie résèque l'ensemble des tissus infectés au niveau des cavités sinusiennes et de la base du crâne. Les mucormycoses ROC représentent une maladie rare mais grave dont la prise en charge chirurgicale doit être connue pour tenter d'améliorer le contrôle de la maladie et la survie des patients.

Par ailleurs, la problématique du traitement des mucormycoses ROC se heurte à plusieurs difficultés:

- Le diagnostic est souvent fait avec retard. En effet, les symptômes initiaux sont aspécifiques et le diagnostic fait par un prélèvement biopsique. - En raison de l'ischémie et de la nécrose, les traitements par voie systémique ont une mauvaise diffusion locale dans la muqueuse infectée. - L'infection s'étend au-delà de la muqueuse. Les filaments fongiques ont une propension à infiltrer la muqueuse, la graisse, les muscles et l'os sous-jacent.

- La tomodensitométrie sous-estime l'étendue de l'infiltration profonde et peut être faussement rassurante au début de la maladie, contribuant ainsi au retard diagnostic.

- Les mucormycoses restent une maladie rare mais grave.

- Il n'existe pas de staging radiologique précis des mucormycoses ROC. Le but de cet article est donc (1) de rappeler les mécanismes d'extension de la maladie, et (2) de montrer l'intérêt et les modalités du traitement chirurgical en fonction de l'extension de la maladie.

\section{Physiopathologie et voies d'extension des mucormycoses ROC}

L'extension des mucormycoses ROC suit un schéma bien connu. Après inhalation des spores, l'infection débute au niveau des cavités 


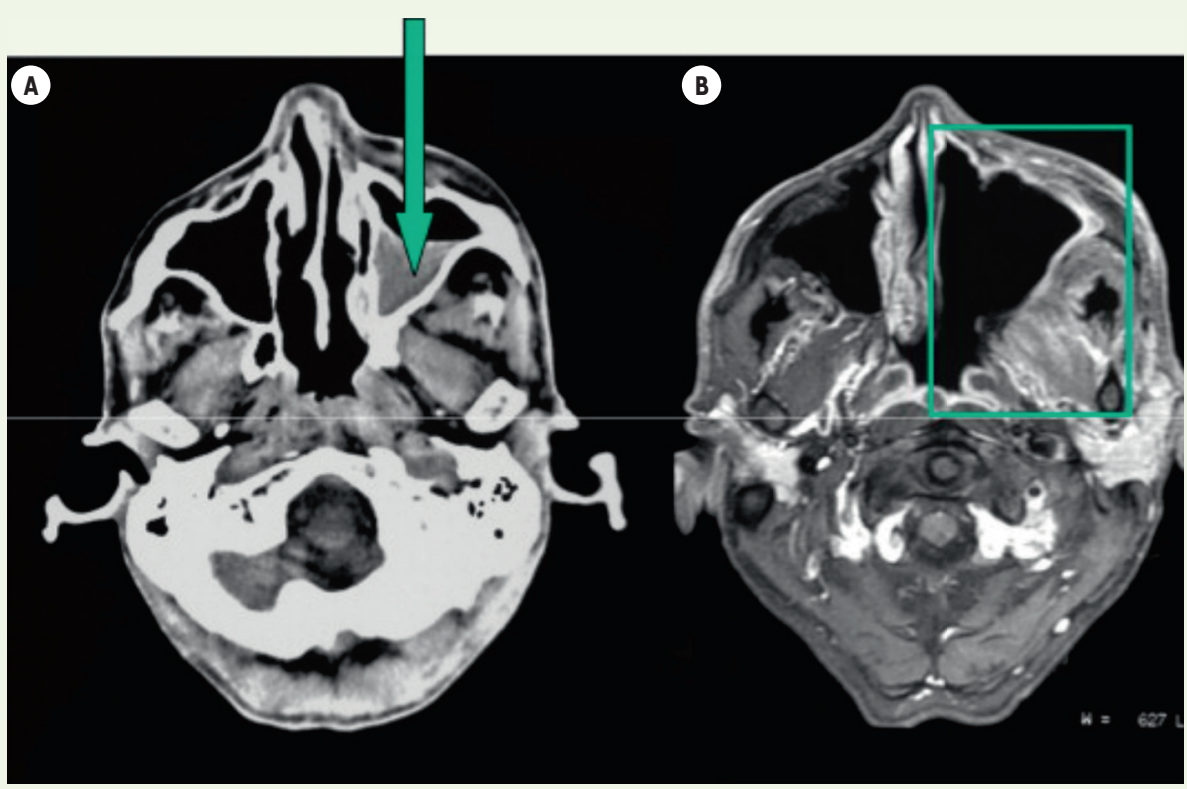

Figure 1. Mucormycose du sinus maxillaire gauche traitée par maxillectomie médiale et abord de la fosse infra-temporale. A. Tomodensitométrie, coupe axiale (la flèche verte indique le sinus touché). B. IRM, coupe axiale, séquence $\mathrm{Tl}$ [19].

ptérygo-palatine comme un témoin de la progression de l'infection. Les rapports anatomiques dans cette région expliquent, en effet, l'importance de son atteinte. La fissure orbitaire inférieure la relie à l'espace rétrobulbaire, la fissure ptérygo-maxillaire à la fosse infra-temporale et

nasales. L'inefficacité des polynucléaires et des macrophages à éliminer les Mucorales et à stopper la germination des spores permet la progression vers les tissus environnants. Ainsi, de la muqueuse des cornets et du septum les lésions se propagent vers les sinus paranasaux [11].

Des zones de nécrose apparaissent au niveau de la muqueuse septale et turbinale. II s'ensuit un franchissement muqueux de l'infection fongique puis une invasion vasculaire. La pénétration à travers l'endothélium vasculaire entraîne alors une thrombose et des lésions de nécrose [12].

L'évolution se fait de proche en proche. Une atteinte de la muqueuse du sinus maxillaire puis du sinus ethmoïdal et, par le trajet des artères et des veines ethmoïdales, l'infection s'étend vers la cavité orbitaire puis vers le sinus caverneux. L'extension intracrânienne peut aussi se faire par le trajet des filets olfactifs à travers la lame criblée [11, 13]. La progression des lésions peut également se faire au niveau du plancher des fosses nasales vers le palais par la formation d'une ulcération transfixiante. Le signe clinique d'escarre noir de la muqueuse du palais est un signe classiquement décrit et témoigne déjà d'une infection étendue et grave.

L'atteinte de la paroi postérieure du sinus maxillaire donne accès par contiguïté à la fosse ptérygo-palatine et à la fosse infra-temporale. L'extension à ces espaces profonds de la face réalise une autre voie d'accès à l'encéphale par les différents foramens de la base du crâne. Hosseini et al., dans une série de 10 cas de mucormycoses rhinocérébrales, donne à la fosse ptérygo-palatine un véritable rôle de « réservoir » de Mucorales. II insiste sur l'importance de l'atteinte de cette zone, la considérant comme un élément clé de la physiopathologie de diffusion de la maladie [13]. Même chez les patients ne présentant pas d'atteinte macroscopique de la paroi postérieure du sinus maxillaire, il retrouve des Mucorales en quantité importante et des tissus nécrosés. II positionne l'envahissement de la fosse à l'espace masticateur, le foramen sphéno-palatin à la cavité nasale, le foramen rotundum et la canal ptérygoïde à la fosse cérébrale moyenne et le canal grand palatin au palais [13]. Les rapports anatomiques de cette fosse ptérygo-palatine justifient le contrôle chirurgical de cette région afin d'éviter des extensions torpides de la maladie.

\section{Traitement chirurgical : intérêt et modalités}

\section{Justification physiopathologique}

La justification physiopathologique du traitement chirurgical repose sur plusieurs points:

- les mucormycoses sont responsables par leur angiotropisme de lésions d'ischémie et de nécrose rendant certaines zones tissulaires inaccessibles aux traitements médicamenteux antifongiques ;

- l'atteinte est intra-muqueuse, intra-osseuse, vasculaire ou nerveuse. Le risque d'extension vers l'orbite ou l'endocrâne est présent tant que l'infection n'est pas contrôlée au niveau sinusien ;

- certaines zones anatomiques constituent de véritables réservoirs de Mucorales qu'il faut contrôler afin de stopper l'extension de la maladie et obtenir le contrôle local de la maladie. La fosse ptérygo-palatine est considérée comme zone clé dans l'extension de la maladie vers l'orbite ou l'endocrâne [13].

\section{Données cliniques}

Dans la littérature, les meilleurs chiffres de survie sont obtenus lorsque la chirurgie a été effectuée chez plus 
de $75 \%$ des patients. II existe une mortalité supérieure en cas de prise en charge médicale seule [3-6]. Blitzer et al. ont rapporté une augmentation de survie de $57,5 \%$ à $79 \%$ en cas de chirurgie radicale [10]. En l'absence de traitement chirurgical, l'état général était jugé précaire ne permettant pas aux patients de supporter une chirurgie radicale $[3-5,7,9]$.

\section{Modalités du traitement chirurgical}

Les modalités du traitement dépendent du niveau et de la sévérité de l'atteinte du patient. Une exérèse large et radicale des lésions doit être réalisée avec résection endobuccale et exentération si nécessaire.

Les voies d'abord externes ont été privilégiées durant de nombreuses années car elles permettaient une résection complète des lésions nécrotiques avec un contrôle optimal [7]. Cependant, la prise en charge des mucormycoses rhinosinusiennes a pu profiter du développement de la chirurgie endoscopique par voie endonasale. La chirurgie endoscopique, initialement développée pour la chirurgie fonctionnelle des sinus puis pour la chirurgie des tumeurs nasosinusiennes et des lésions de la base du crâne, est également utilisée dans le cadre des mucormycoses ROC. Le but n'est pas de réaliser une chirurgie a minima, mais d'obtenir le même résultat qu'avec une chirurgie par voie externe en ayant de meilleures suites postopératoires. II s'agit de réaliser l'exérèse de la totalité des tissus nécrotiques avec un bénéfice esthétique et fonctionnel.

Les possibilités de la chirurgie sinusienne endoscopique sont très larges et permettent de réaliser une résection complète des tissus nécrosés dans de nombreux cas [7].

Dans les formes suspectées précocement de mucormycoses rhinosinusiennes, les atteintes initiales touchent les fosses nasales puis les sinus ethmoïdaux, sphénoïdaux et maxillaires [7]. Ces atteintes peuvent être traitées par voie endonasale par la réalisation d'ethmoïdectomie antéro-postérieure, de sphénoïdotomie ou de maxillectomie médiane.

Pour des lésions plus étendues, de larges résections peuvent être réalisées (Figure 1). Si nécessaire, une résection dure-mérienne avec reconstruction de l'étage antérieur peut également être réalisée par voie endonasale (voie transcribriforme), comme dans la chirurgie des tumeurs de la base du crâne.

Ces techniques de chirurgie endonasale par voie endoscopique permettent: (1) un bilan lésionnel complet; (2) un diagnostic précoce [7]; (3) une évaluation précise de l'étendue de l'infection avec prélèvements locaux; et (4) la possibilité de répéter les interventions chirurgicales pour le contrôle de la maladie.

Plusieurs études rapportent l'intérêt des examens anatomopathologiques extemporanés pour guider l'étendue de la résection chirurgicale $[8,14,15]$. La coloration par Calcofluor a notamment été utilisée pour analyser des marges de résection en améliorant la détection de tissus atteints par l'infection [16].

Dans les formes plus étendues (Figure 2), des abords externes tels que des résections cutanées, des résections palatines et l'exentération orbitaire peuvent être associées à la chirurgie endonasale.

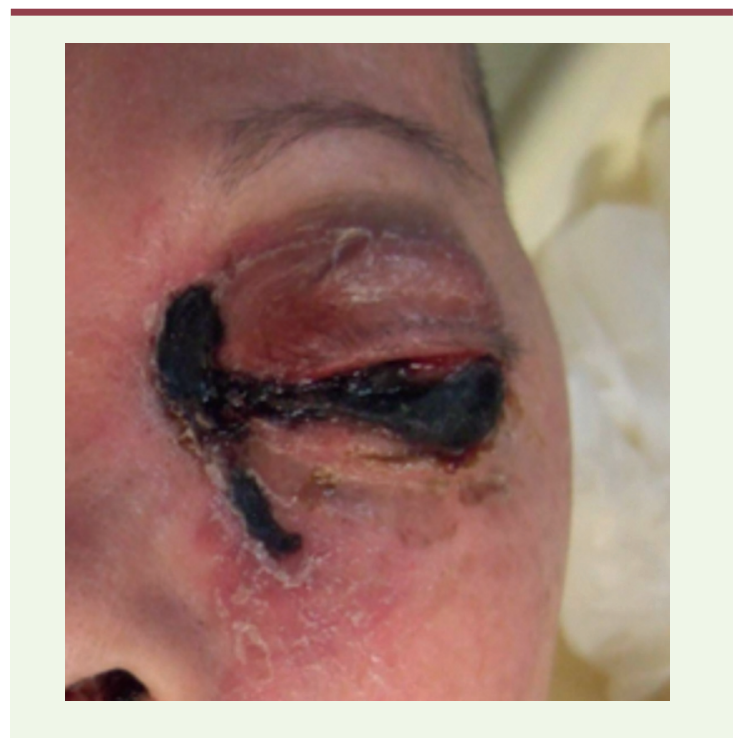

Figure 2. Aspect de nécrose palpébrale inférieure et canthale interne gauche marquant le stade avancé de l'atteinte orbitaire.

En effet, le traitement des mucormycoses ROC peut justifier une prise en charge chirurgicale agressive avec possiblement une exentération orbitaire (Figure 3) en cas d'atteinte orbitaire irréversible. Cette chirurgie est indiquée pour prévenir l'extension intracérébrale et tenter d'améliorer le pronostic vital qui est en jeu. Récemment, certains auteurs se sont interrogés sur sa nécessité, étant donné le caractère mutilant de l'exentération. En effet lorsque lorsqu'un patient atteint de mucormycose rhinosinusienne ne bénéficie pas d'une exentération et ne survit pas à l'infection, on peut se demander si l'exentération aurait pu le sauver. Inversement, lorsqu'un patient survit mais qu'une exentération a été réalisée, on peut se demander si elle était réellement nécessaire pour sa survie. C'est pourquoi certains auteurs ont tenté d'établir des indications pour l'exentération. Nithyanandam et al. [3] rappellent que l'orbite peut être épargnée notamment lorsque les facteurs favorisants peuvent être corrigés rapidement, même en présence d'une ophtalmoplégie complète et d'une occlusion de l'artère centrale de la rétine. Un traitement conservateur doit également être envisagé pour une atteinte isolée d'un muscle extra-oculaire avec une vision préservée et une absence de progression. Blitzer et al. [10] préconisent une exentération orbitaire en cas de nécrose de l'apex. Cliniquement, une atteinte de l'apex orbitaire se manifeste par un syndrome (SAO : syndrome de l'apex orbitaire) comprenant une ophtalmoplégie, un ptosis, une diminution de la sensibilité cornéenne et une baisse d'acuité visuelle. II peut être dû à une compression mécanique de la région, une vascularite ou une ischémie de la région de l'apex 


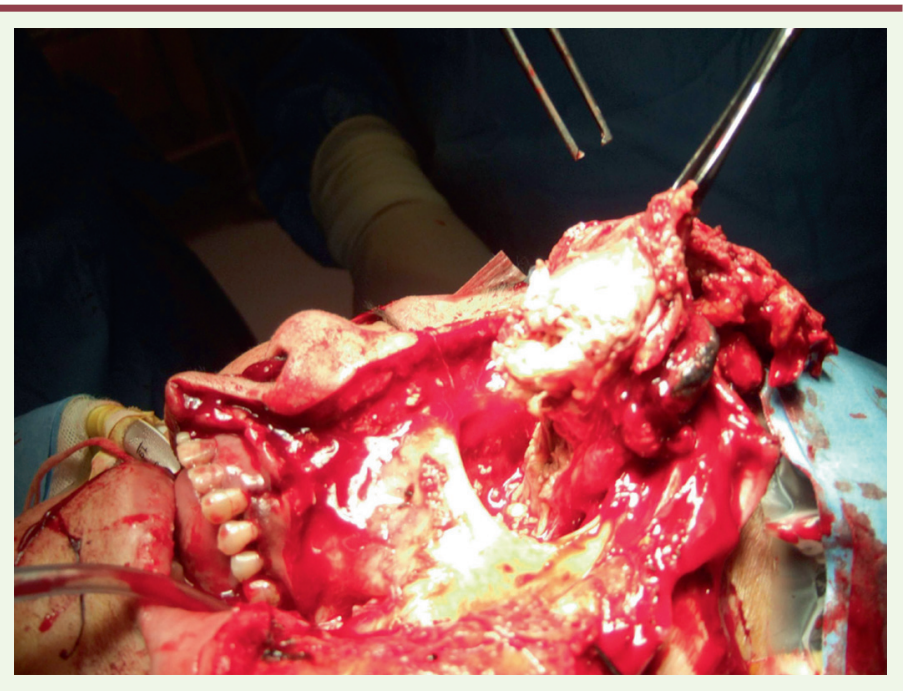

Figure 3. Exentération orbitaire chez un patient présentant une mucormycose rhino-orbitaire.

de l'orbite. Pour Peterson, l'indication d'exentération orbitaire dépend de l'agressivité de la présentation, du type de facteur favorisant et de la réponse initiale au traitement. Dans certains cas, l'orbite peut être préservée notamment dans les présentations moins agressives [9]. Selon O'neil et al., l'exentération ne doit être envisagée que pour une infection active rhino-orbitaire une fois que l'extension cérébrale survient [17]. Hargrove et al. [18], dans une revue de la littérature des cas d'exentérations pour les mucormycoses rhinosinusiennes, a tenté d'établir des indications pour ce geste. L'étude des 292 cas retrouvés n'a pas permis de déterminer une réelle conduite à suivre. L'indication dépend du jugement des praticiens prenant en charge le patient [18] et du pronostic vital évalué à court terme.

\section{Intérêt du traitement chirurgical radical sur la survie}

Dans le cadre d'une étude des 22 cas de mucormycoses ROC issus de l'étude Rétrozygo [2], un staging de la prise en charge chirurgicale a été établi et la survie des patients en fonction du type de traitement a été étudiée.

Le type d'intervention chirurgicale a été défini comme suit :

- Traitement chirurgical grade I : prélèvement biopsique, chirurgie diagnostique, absence de geste d'exérèse.

- Traitement chirurgical grade $\|$ : exérèse simple des lésions nécrotiques par voie endonasale.

- Traitement chirurgical grade III : exérèse étendue avec résection base du crâne, résection endocrânienne, résection de paroi interne orbite, exentération, résection endobuccale.

La survie globale était de $57 \%$ (13/22) avec un suivi moyen de 40,3 mois. II existait une différence significative de survie en fonction du type de traitement chirurgical effectué (Figure 4). La survie actuarielle était respectivement de $80 \%, 14 \%$ et $80 \%$ pour les patients ayant eu un traitement de grade I (simple biopsie), II (résection des tissus nécrosés) et III (chirurgie radicale). La survie des patients ayant eu un traitement chirurgical radical (grade III) était respectivement de $90 \%$ et $80 \%$ à 6 mois et 1 an alors qu'elle était de $0 \%$ à 6 mois et 1 an pour ceux ayant eu un geste plus limité (grade II) $(p<0,05)$ (Vironneau et al., données soumises pour publication). Ces résultats suggèrent que la chirurgie radicale a l'intérêt d'enlever des zones nécrotiques et les tissus sous-jacents ischémiques, difficilement accessibles aux antifongiques.

Tous les patients ayant eu un traitement chirurgical radical avaient une atteinte radiologique orbitaire et/ ou cérébrale plus ou moins étendue suggérant le bénéfice de la résection sinusienne étendue en conjonction avec le traitement médical, sans nécessairement réaliser d'exentération ou de résection de la dure mère. Le chirurgien peut réaliser une résection radicale par voie endonasale sans nécessairement avoir recours à une chirurgie mutilante. L'étendue de la résection est adaptée aux constatations peropératoires ce d'autant que l'évaluation radiologique préopératoire ne permet pas toujours de distinguer les zones nécrotiques des zones infectées. Ces résultats méritent cependant une validation dans le cadre d'une étude prospective (PHRC national MICCA). En effet, le succès d'un traitement de ces affections graves repose vraisemblablement sur plusieurs éléments : diagnostic précoce, correction des facteurs prédisposants, résection chirurgicale étendue, thérapie antifongique à forte dose instaurée rapidement. Ce protocole MICCA comporte, en association au traitement antifongique par amphotéricine B liposomale, une ou plusieurs chirurgie(s) de révision adaptées aux prélèvements anatomopathologiques et mycologiques afin d'augmenter le contrôle local de la maladie et la survie des patients.

\section{Conclusion}

Les mucormycoses ROC représentent une pathologie infectieuse et nécrosante grave au niveau sinusien, orbitaire et cérébral dont le pronostic est largement conditionné par la rapidité et l'efficacité de la prise en charge tant médicale que chirurgicale. Une chirurgie radicale d'exérèse des lésions nécrotiques par voie endonasale permet l'obtention du contrôle local de la maladie. Des abords externes peuvent être nécessaires (résection cutanée, résection endobuccale, exentération). L'indication de ces procédures chirurgicales doit être évaluée en fonction de l'évolution des lésions. Plusieurs chirurgies peuvent être nécessaires pour le contrôle local de la maladie. Les séquelles fonctionnelles de ces gestes chirurgicaux peuvent être graves. Leur justification est fondée sur l'amélioration du pronostic vital. $\diamond$ 


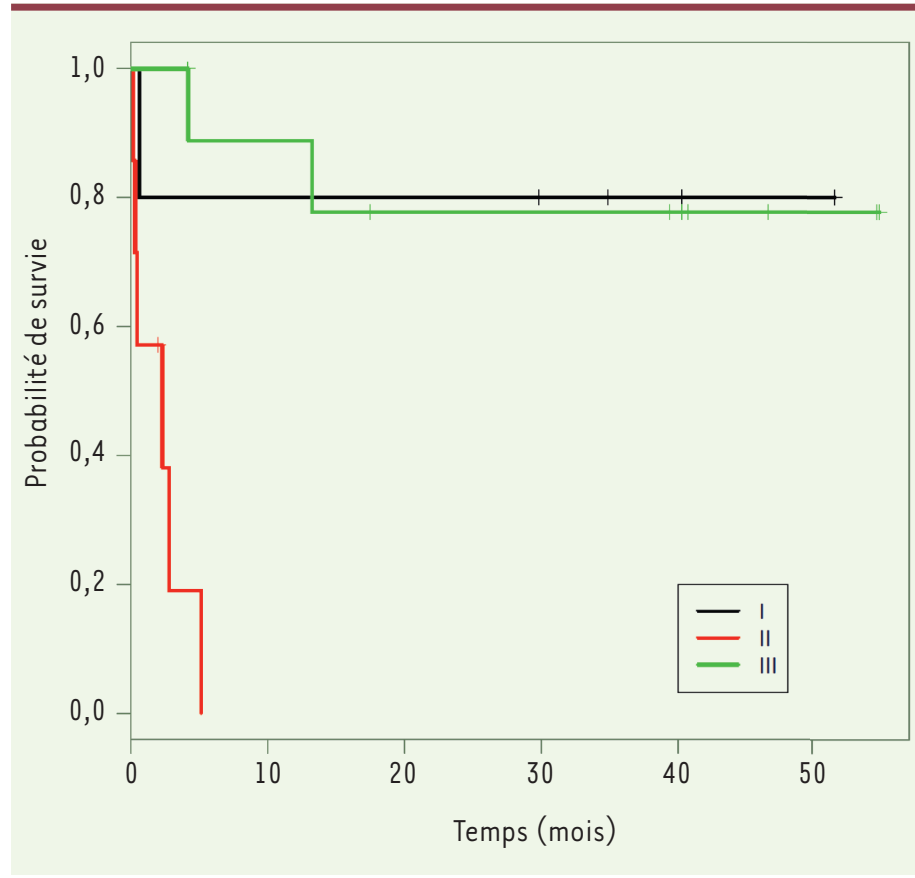

Figure 4. Courbe de Kaplan-Meier montrant la survie des patients en fonction du type de traitement chirurgical.

\section{SUMMARY}

Rhino-orbito-cerebral mucormycosis, surgical treatment, state of the art

Rhino-orbito-cerebral mucormycoses constitute a severe fungal infection. These infections mostly arise in immunosuppressed patients. The surgery aiming at resecting necrosed hurts showed its interest in term of survival for lung and cutaneous mucormycosis. However, treatment of rhino-orbito-cerebral location of mucormycosis is not well defined. Transnasal endoscopic surgery allows local control of the disease, better post-operative outcomes than transfacial approaches and less sequelae. However, transfacial approaches are sometimes necessary to allow cutaneous resection or exenteration, the indications of which still remain controversial. The retrospective study of 22 patients with mucormycosis allowed to show that radical surgical treatment allowed local control of the disease with an improved survival. Further prospective studies (PHRC MICCA, current) are required to standardize the management of this rare but potentially lethal pathology. $\diamond$

\section{LIENS D'INTÉRÊT}

Les auteurs déclarent n'avoir aucun lien d'intérêt concernant les données publiées dans cet article.

\section{RÉFÉRENCES}

1. Roden MM, Zaoutis TE, Buchanan WL, et al. Epidemiology and outcome of zygomycosis: a review of 929 reported cases. Clin Infect Dis 2005 ; 41 : 634-53.

2. Lanternier F, Dannaoui $\varepsilon$, Morizot G, et al. A Global Analysis of Mucormycosis in France: The RetroZygo Study (2005-2007). Clin Infect Dis 2012 ; 54 (suppl 1) : S35-43.

3. Nithyanandam S, Jacob MS, Battu RR, et al. Rhino-orbito-cerebral mucormycosis. A retrospective analysis of clinical features and treatment outcomes. Indian J Ophthalmol $2003 ; 51: 231-6$.

4. Mohindra S, Gupta R, Bakshi J, Gupta SK. Rhinocerebral mucormycosis: the disease spectrum in 27 patients. Mycoses $2007 ; 50: 290-6$.

5. Talmi YP, Goldschmied-Reouven A, Bakon M, et al. Rhino-orbital and rhinoorbito-cerebral mucormycosis. Otolaryngol Head Neck Surg 2002; 127 : 22-31.

6. Munir N, Jones NS. Rhinocerebral mucormycosis with orbital and intracranial extension: a case report and review of optimum management. J Laryngol Otol $2007 ; 121: 192-5$.

7. Jiang RS, Hsu Cy. Endoscopic sinus surgery for rhinocerebral mucormycosis. Am J Rhinol $1999 ; 13: 105-9$.

8. Reed C, Bryant R, Ibrahim AS, et al. Combination polyene-caspofungin treatment of rhino-orbital-cerebral mucormycosis. Clin Infect Dis 2008; 47 : 364-71.

9. Peterson KL, Wang M, Canalis RF, Abemayor $\varepsilon$. Rhinocerebral mucormycosis: evolution of the disease and treatment options. Laryngoscope 1997 ; 107 : 855-62.

10. Blitzer A, Lawson W, Meyers BR, Biller HF. Patient survival factors in paranasal sinus mucormycosis. Laryngoscope $1980 ; 90: 635-48$.

11. Dan M. Mucormycosis of the head and neck. Curr Infect Dis Rep 2011 ; 13 : 123-31.

12. Spellberg B, Edwards J Jr, Ibrahim A. Novel perspectives on mucormycosis: pathophysiology, presentation, and management. Clin Microbiol Rev 2005 ; $18: 556-69$.

13. Hosseini SM, Borghei P. Rhinocerebral mucormycosis: pathways of spread. Eur Arch Otorhinolaryngol $2005 ; 262$ : 932-8.

14. Hofman V, Castillo L, Betis F, et al. Usefulness of frozen section in rhinocerebral mucormycosis diagnosis and management. Pathology 2003; $35: 212-6$.

15. Langford JD, McCartney DL, Wang RC. Frozen section: guided surgical debridement for management of rhino-orbital mucormycosis. Am J Ophthalmol $1997 ; 124: 265-7$.

16. McDermott NE, Barrett J, Hipp J, et al. Successful treatment of periodontal mucormycosis: report of a case and literature review. Oral Surg Oral Med Oral Pathol Oral Radiol Endod 2010 ; 109 : e64-9.

17. O’Neill BM, Alessi AS, George EB, Piro J. Disseminated rhinocerebral mucormycosis: a case report and review of the literature. J Oral Maxillofac Surg $2006 ; 64: 326-33$.

18. Hargrove RN, Wesley RE, Klippenstein KA, et al. Indications for orbital exenteration in mucormycosis. Ophthal Plast Reconstr Surg 2006 ; 22 : 286 91.

19. Meas T, Mouly S, Kania R, et al. Zygomycosis: an uncommon cause for peripheral facial palsy in diabetes. Diabetes Metab 2007 ; $33: 227-9$.

20. Goyal P, Leung MK, Hwang PH. Endoscopic approach to the infratemporal fossa for treatment of invasive fungal sinusitis. Am J Rhinol Allergy 2009; $23: 100-4$.

\section{TIRÉS À PART}

R. Kania 